\title{
Chapter 6 \\ Check List for Trocar-Based Surgeries of Anterior Segment
}

Trocar surgery is best learned in a stepwise manner. Start to perform surgeries with one trocar and proceed later on with two trocars. The following checklist may be a guide for your surgical development:

\section{How to Learn Trocar Surgery for an Anterior Segment Surgeon in a Stepwise} Manner. See the Following Check List

1. Insertion and removal of one trocar on the temporal side.

2. Anterior vitrectomy through trocar cannula.

3. Master SICS.

4. Elevation of anterior dislocated nucleus.

5. Elevation of anterior dislocated IOL.

6. Secondary IOL implantation.

7. Insertion of two trocars on the temporal side. One trocar for irrigation line.

8. Anterior vitrectomy from pars plana with infusion line.

Start trocar surgery with steps 1-2 and get acquainted to this new technique. The next steps require that you master SICS (step 3). Then try to elevate a subluxated nucleus, extract it through a scleral tunnel and then continue with an anterior vitrectomy through trocar cannulas (step 4). Similarly, you can elevate and extract an anterior dislocated IOL and perform a secondary IOL implantation (steps 5-6). The next big step is the insertion of two trocar cannulas. The second trocar cannula is used for the infusion (=irrigation line). The irrigation line gives you two free hands. Perform an anterior vitrectomy and all other surgeries with two trocars (steps 7-8). It is important to get acquainted to two trocars before you proceed to posterior segment procedures. 